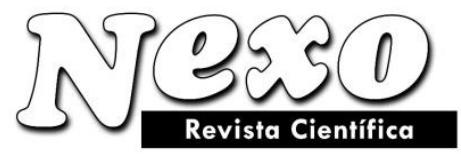

ISSN-L 1818-6742

Impreso en Nicaragua.

http://revistasnicaragua.peri.net.ni/index.php/nexo/index

Vol. 24, No. 02, pp.123-129/Diciembre 2011

\title{
Interfaz Gráfica Computacional para Destilación Multicomponente utilizando Métodos Cortos
}

\author{
G. Domínguez Hernández, I. Sánchez Bazán, A. Osorio Mirón, G. E. González Landero, L. A. \\ Sánchez Bazán, E. Hernández Aguilar. \\ Facultad de Ciencias Químicas, Universidad Veracruzana \\ Prolong. Oriente 6 No. 1009, Col Rafael Alvarado C. P. 94340 Orizaba, Veracruz, México. \\ (goya_as, bazaine24)@hotmail.com, anosorio@uv.mx
}

(Recibido/received: 12-Octubre-2011; aceptado/accepted: 09-Diciembre-2011)

\begin{abstract}
RESUMEN
En este trabajo, se elaboró una interfaz gráfica que determina por medio de simulación en estado estacionario el número de platos necesarios en el proceso de destilación multicomponente, así como la composición en el flujo de destilado y fondos de una torre de destilación utilizando métodos cortos. La interfaz gráfica de usuario, se construyó en el lenguaje de programación Python 2.5, permite presentar los datos de entrada y salida para el caso de columnas de destilación operando en régimen permanente. Los resultados del uso de la interfaz gráfica muestran una forma simple de realizar diseños preliminares de torres de destilación y su potencialidad como material de apoyo para el estudio y comprensión de conceptos básicos en destilación multicomponente. En un trabajo posterior se podrá visualizar el desempeño del modelo dinámico a través de perturbaciones programadas en las condiciones de operación.
\end{abstract}

Palabras claves: destilación multicomponente, métodos cortos, interfaz gráfica de usuario, Phyton 2.5.

\begin{abstract}
In this work, we developed a graphical interface that determines by steady-state simulation the number of plates required for multicomponent distillation process and the composition of distillate and bottoms flow of a distillation tower using short-cut methods. The graphical user interface was built in the programming language Python 2.5 , allows to present the input and output data for the case of distillation columns operating in steady state. The results of using the graphical interface shows a simple way to make preliminary designs of distillation towers and its potential as support material for the study and understanding of basic concepts in multicomponent distillation. In a later work it will can display the dynamic model performance through scheduled disturbances in operating conditions.
\end{abstract}

Keywords: multicomponent distillation, shorcut methods, graphical user interface, Phyton 2.5.

\footnotetext{
* Autor para la correspondencia
} 


\section{INTRODUCCIÓN}

La destilación es unos de los métodos de separación mas comúnmente usados en la industria química, de tal forma que para el cálculo y diseño de columnas de destilación se han empleado diversas herramientas para determinar el número de platos y el tamaño de la columna. Dependiendo del número de componentes en la mezcla a separar, la destilación se denomina binaria o multicomponente, y se emplean diferentes métodos de cálculo. Para mezclas multicomponentes, la separación de los componentes depende de la distribución de las sustancias entre la fase gaseosa y la fase líquida; se aplica a los casos en los que todos los componentes están presentes en las dos fases. La nueva fase se crea por evaporación o condensación a partir de la solución original (King, 1981; Kister, 1992). En este caso se emplean métodos tales como los de Thiele-Geddes, Lewis-Mathewson y $\Phi$, combinando las ecuaciones de Kirkbride, Underwood, Fenske y el diagrama de ErbarMaddox (KUFEM) para calcular el número de platos teóricos y determinar la localización del plato de alimentación, esta combinación facilita el cálculo de los componentes más importantes de una columna de destilación simple, con una alimentación y dos salidas (Chang, 1980; Dimitrios, 1981).

Puesto que se desea obtener el número de etapas necesarias para conseguir la separación especificada y conocer la posición óptima del plato de alimentación, una vez definidas las variables y los parámetros caracteristicos del proceso de separación, a partir de los valores supuestos para la recuperación del componente clave $(L K)$ ligero en el destilado y el componente clave pesado $(H K)$ en el fondo, se sigue una secuencia de cálculo como la que se muestra en las referencias (Chang, 1980; Dimitrios, 1981). Esta secuencia se codificó en el lenguaje de programación Python 2.5 y se construyó una interfaz gráfica computacional que permite presentar los datos de entrada y visualizar los resultados numéricos (datos de salida) en el cálculo de columnas de destilación en estado estacionario. Los resultados muestran una forma rápida y simple de realizar cálculos para el diseño preliminar de equipo de destilación, además este simulador sirve como material didáctico de apoyo para la comprensión de conceptos básicos en separaciones multicomponentes.

\section{METODOLOGÍA}

Una torre de destilación que está compuesta de las secciones de rectificación (arriba de la alimentación) y de agotamiento (debajo de la alimentación) es capaz de lograr una separación más o menos fina entre productos o componentes puros de la mezcla, es decir, entre los componentes clave ligero y pesado. El clave ligero es el componente más volátil cuya concentración será controlada en el producto del fondo y el clave pesado es el componente menos volátil cuya concentración será controlada en el producto del domo.

La solución numérica del modelo para el cálculo del número de etapas y la localización de la etapa de alimentación se realiza comunmente empleando el método de Newton-Raphson multivariable (Schmitz Abe, 1999; Monroy-Loperena, 2003). Primero, se especifican las variables necesarias para caracterizar el flujo de alimentación (composición y valores promedio de volatilidades relativas), el porcentaje de recuperación del componente clave ligero $(L K)$ en el destilado y del componente clave pesado $(H K)$ en el residuo, la relación de reflujo inicial, y se suponen valores para la recuperación de los componentes clave, ligero en el destilado y pesado en el fondo. Después, se realizan los pasos siguientes (Chang, 1980; Al-Tuwain y Luyben, 1991; Abad-Zárate et al, 2006).

1. Se calcula el número de moles de los componentes clave en el destilado y en el residuo con las ecuaciones (1-4).

$$
\begin{aligned}
& d_{L k}=\left(d_{L K} / f_{L K}\right)\left(f_{L K}\right) \\
& b_{L K}=f_{L K} \quad d_{L K} \\
& d_{H K}=\left[\begin{array}{ll}
1 & b_{H K} / f_{H K}
\end{array}\right]\left(f_{H K}\right) \\
& b_{H K}=f_{H K} \quad d_{H K}
\end{aligned}
$$

Para los componentes no claves, se utiliza la ecuación de Hengstebeck-Geddes (5),

$$
\ln \left(d_{i} / b_{i}\right)=C_{1}+C_{2} \ln
$$

o en la forma de la ecuación (6).

$$
\frac{d_{i}}{b_{i}}=\exp \left(C_{1}+C_{2} \ln \quad i\right)
$$

donde,

$$
\begin{aligned}
& C_{1}=\ln \left(d_{H K} / b_{H K}\right) \\
& C_{2}=\frac{\ln \left[\left(d_{L K} / b_{L K}\right)\left(b_{H K} / d_{H K}\right)\right]}{\ln \propto_{L K}}
\end{aligned}
$$


Aplicando el balance de materia en esta ecuación, se tienen las ecuaciones (7) y (8).

$$
\begin{aligned}
& b_{i}=\frac{f_{i}}{1+\exp \left(C_{1}+C_{2} \ln { }_{i}\right)} \\
& d_{i}=f_{i} \quad b_{i}
\end{aligned}
$$

Las fracciones mol de cada componente en el destilado y en el fondo se calculan con las ecuaciones (9) y (10).

$$
\begin{aligned}
& x_{D_{i}}=\frac{d_{i}}{d_{i}} \\
& x_{B_{i}}=\frac{b_{i}}{b_{i}}
\end{aligned}
$$

Se definen dos variables, una que relaciona el flujo de destilado y el flujo de alimentación del clave ligero, y otra que relaciona el flujo de fondos y el flujo de alimentación del clave pesado,

$$
s_{1}=d_{L K} / f_{L K} \quad s_{2}=b_{H K} / f_{H K}
$$

Enseguida, se establecen dos funciones, (11) y (12).

$$
\begin{aligned}
& g_{\mathbf{1}}\left(s_{1}, s_{2}\right)=\left(x_{D_{H K}}\right)_{c a}-x_{D_{H K}} \\
& g_{2}\left(s_{1}, s_{2}\right)=\left(x_{B_{L K}}\right)_{c a}-x_{B_{L K}}
\end{aligned}
$$

que dependen de las dos variables definidas antes. En este punto, se tiene un sistema de ecuaciones algebraicas no lineales, cuya solución se obtiene aplicando el método de Newton-Raphson multivariable. Los valores de las dos variables definidas, $s_{1}$ y $s_{2}$, se encuentran cuando las dos funciones establecidas, $g_{1}$ y $g_{2}$, se aproximan a cero.

2. Se calcula el número mínimo de platos usando la ecuación de Fenske (13).

$$
N_{\min }=\frac{\ln \left[\left(x_{D_{L K}} / x_{B_{L K}}\right)\left(x_{B_{H K}} / x_{D_{H K}}\right)\right]}{\ln L K}
$$

3. Cálculo del reflujo mínimo usando la ecuación de Underwood (14).

$$
1 q={ }_{1}^{n} \frac{{ }_{i} X_{F_{i}}}{i}
$$

que se resuelve utilizando el método de bisección. El valor obtenido del llamado parámetro de Underwood, se sustituye en la ecuación (15), para obtener el reflujo mínimo.

$R_{\min }+1={ }_{1}^{n} \frac{{ }_{i} X_{D_{i}}}{i}$

4. Cálculo del número de platos teóricos usando el método de Gilliland. El número de platos teóricos, utilizando el reflujo de operación, se obtiene con la correlación de Gilliland, ecuación (16):

$$
Y=1 \quad \exp \left[\frac{(1+54.4 X)(X \quad 1)}{(11+117.2 X) \sqrt{X}}\right]
$$

donde,

$$
X=\frac{R R_{\text {min }}}{R+1} \quad Y=\frac{N N_{\text {min }}}{N+1}
$$

5. Localización del plato de alimentación. Se emplea la ecuación de Kirkbride (17), para determinar la posición del plato de alimentación en la columna.

$$
\log \left(\frac{m}{p}\right)=0.206\left\{\left(\frac{B}{D}\right)\left(\frac{x_{H}}{x_{L}}\right)_{F}\left[\frac{\left(x_{L}\right)_{B}}{\left(x_{H}\right)_{D}}\right]^{2}\right\}
$$

En la Fig. 1, se resume la secuencia de cálculo del método descrito: Fenske-Underwood-GilliandKirkbride, FUGK, a partir de este algoritmo se codifican las ecuaciones del método y se diseña una interfaz grafica de usuario para la presentación de los resultados numéricos.

\section{RESULTADOS Y DISCUSIÓN}

La interfaz gráfica computacional se desarrolló en Python 2.5, el cual es un lenguaje de programación de código abierto, tanto para uso profesional como para el aprendizaje de la programación; es muy expresivo, es decir, los programas codificados en Python son muy compactos, suelen ser bastante más corto que su equivalente en leguaje $C$, y la sintaxis es muy elegante lo que permite una escritura más fácil que en otros programas. Además, permite la ejecución del programa en diferentes sistemas operativos. En la Fig. 1, se presenta el diagrama de flujo para el cálculo por métodos cortos en destilación multicomponente. Los 
módulos de los procedimientos empleados por el método FUGK, permiten el cálculo del número de platos teóricos y la localización del plato de alimentación, utilizando una solución numérica con el método de Newton-Raphson multivariable.

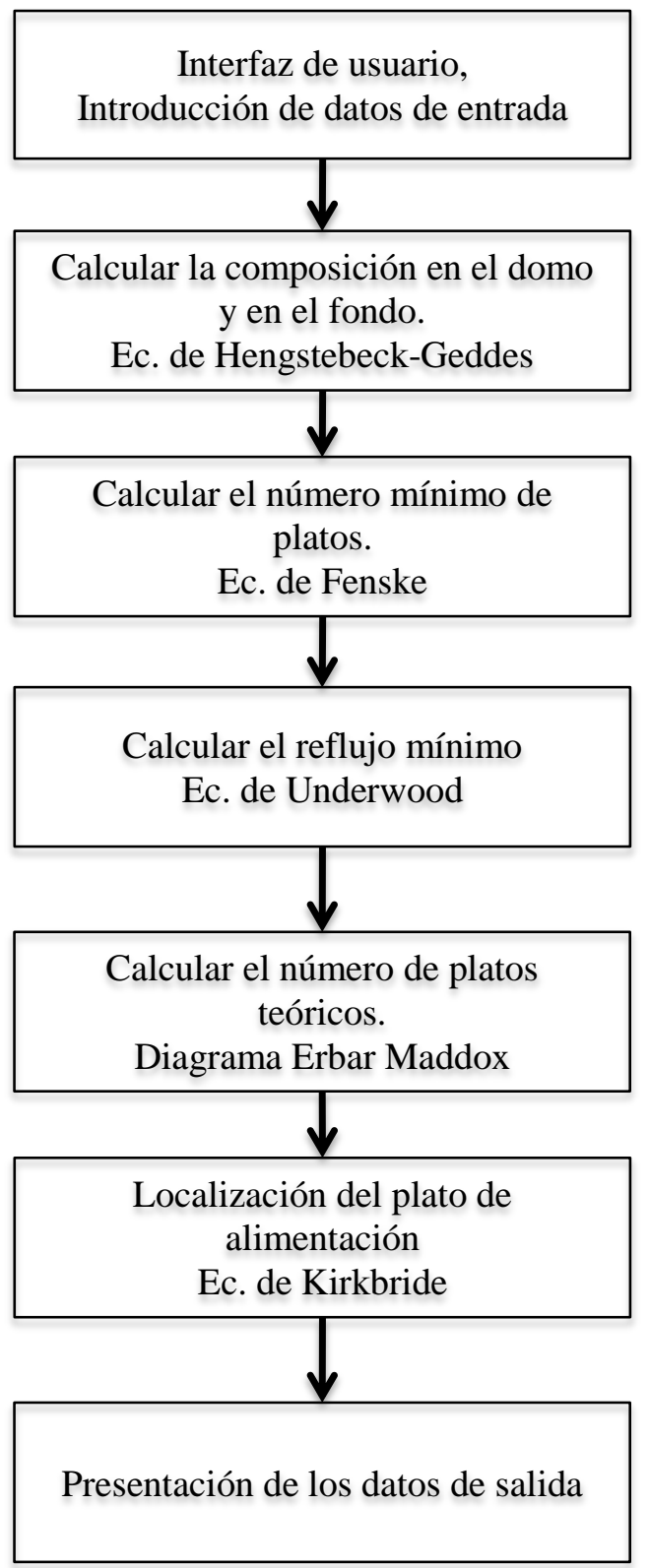

Fig. 1 Secuencia de cálculo, método FUGK.

Se desarrolló una función para cada uno de los pasos descritos en la Fig. 1, estas funciones tienen por nombre: $H G$ para el cálculo de los componentes clave y no clave; $F E$ para el cálculo del número mínimo de platos; UWD para el cálculo del reflujo mínimo; y $G L L D$ para el cálculo del número de platos.
El programa inicia con la función principal llamada Calcular, que toma los valores de las variables de inicio para alimentar las funciones antes descritas y comenzar el cálculo por medio de la función $N e w R$. Los datos que se deben ingresar al programa, y que corresponden a las variables necesarias para las ecuaciones, se pueden visualizar en la Fig. 2, en la sección Datos de entrada. Los datos de entrada corresponden a las variables siguientes: número de componentes de la mezcla (NOC), componente clave ligero $(L K)$, componente clave pesado $(H K)$, condición térmica de la alimentación $(q)$, relación de reflujo de operación (RRM), fracción mol del componente clave pesado en el destilado $(X D H K)$, fracción del componente clave ligero en el fondo $(X B L K)$, composición para cada uno de los componentes de alimentación $(X F(i))$, donde $i=1 \ldots N O C$, volatilidad relativa para cada uno de los componentes de la alimentación, $\mu_{i}$, valores iniciales supuestos de las distribuciones de los componentes, clave ligero en el domo y clave pesado en el fondo.

El programa dispone de valores supuestos iniciales para cada una de estas variables, de tal forma que el usuario pueda correr fácilmente el programa. El botón Calcular (Fig. 2) ejecuta el método corto codificado, primero toma las variables iniciales de entrada, llama las funciones $H G, F E, U W D, G L L D$ y resuelve las ecuaciones respectivas utilizando el método de NewtonRaphson multivariable (función NewR). Ahora el programa devolverá los datos de salida, con la información necesaria proporcionada por el método de cálculo (claramente diferenciados de color rojo, ver Fig. 3).

Los resultados numéricos que se muestran son: la composición de cada uno de los elementos de la mezcla, el balance de materia para las dos salidas (tomando una base de cálculo de 100 moles/h), el número mínimo de platos, el reflujo mínimo, el número de platos teóricos, la posición del plato en el que debe alimentarse la mezcla, el número de platos en la sección de rectificación y el número de platos en la sección de agotamiento.

Una modificación de la interfaz gráfica se presenta en la Fig. 4, que se ha construído con características amigables al usuario, como una contribución a la elaboración de un laboratorio virtual en destilación y evaporación para el autoaprendizaje, y como complemento a experiencias educativas en operaciones unitarias de transferencia de masa y/o procesos de separación. 
Domínguez G. el al

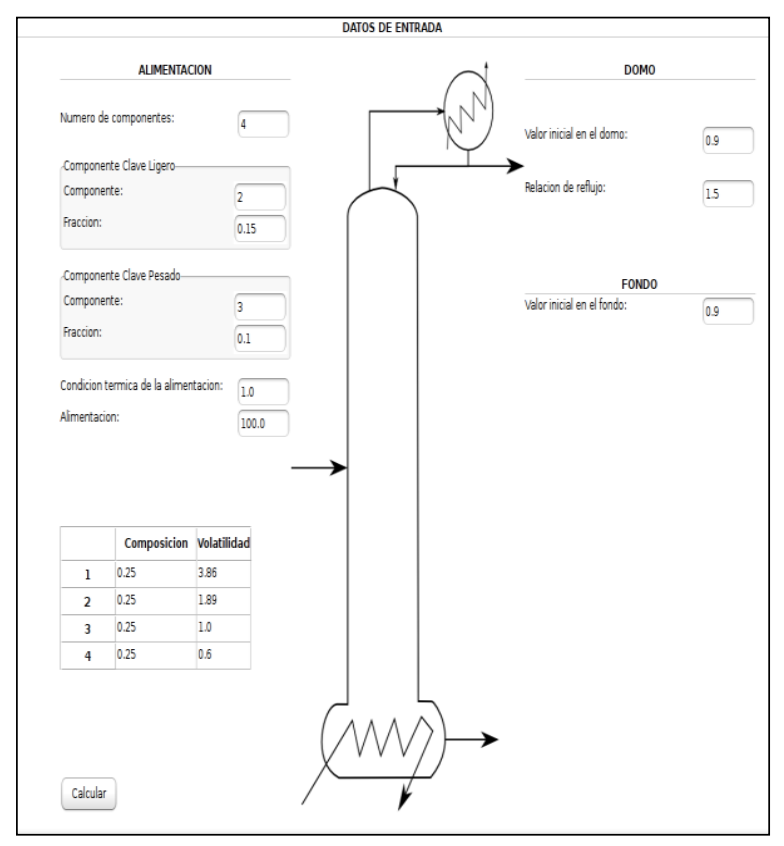

Figura. 2 Interfaz gráfica del programa.

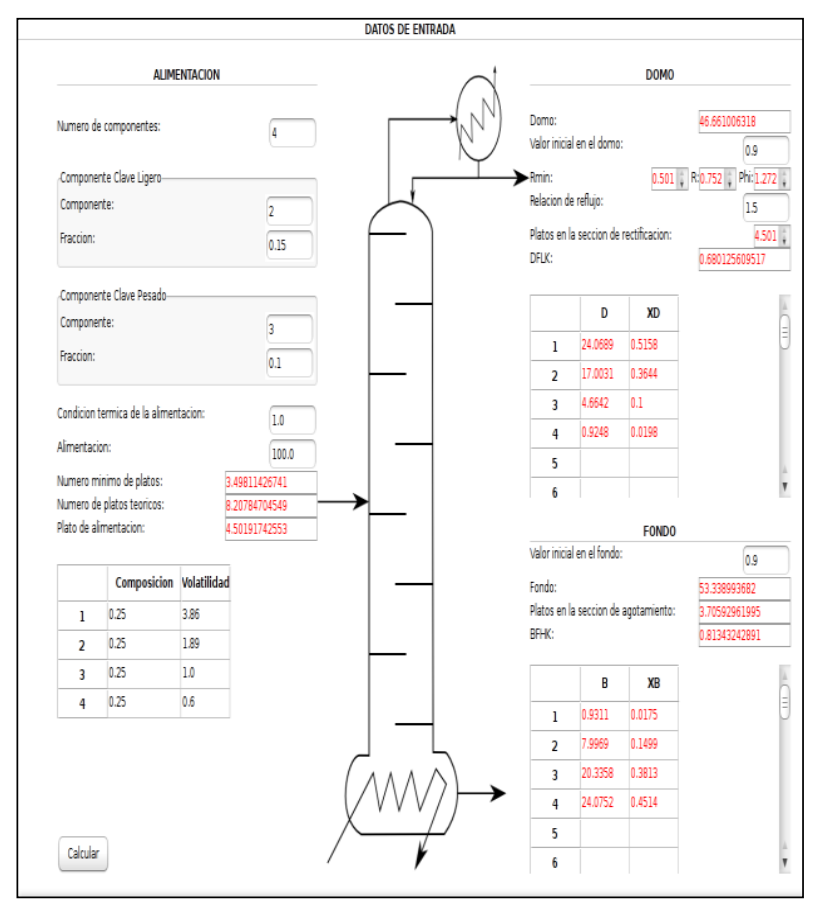

Figura 3 Resultados numéricos.

En la interfaz se realizaron modificaciones con el objeto de tener una acción interactiva para la introducción de datos y la ejecución de los cálculos numéricos, Fig. 5. Los círculos en rojo indican las cajas que debe llenar el usuario con sus datos de entrada supuestos o calculados previamente.

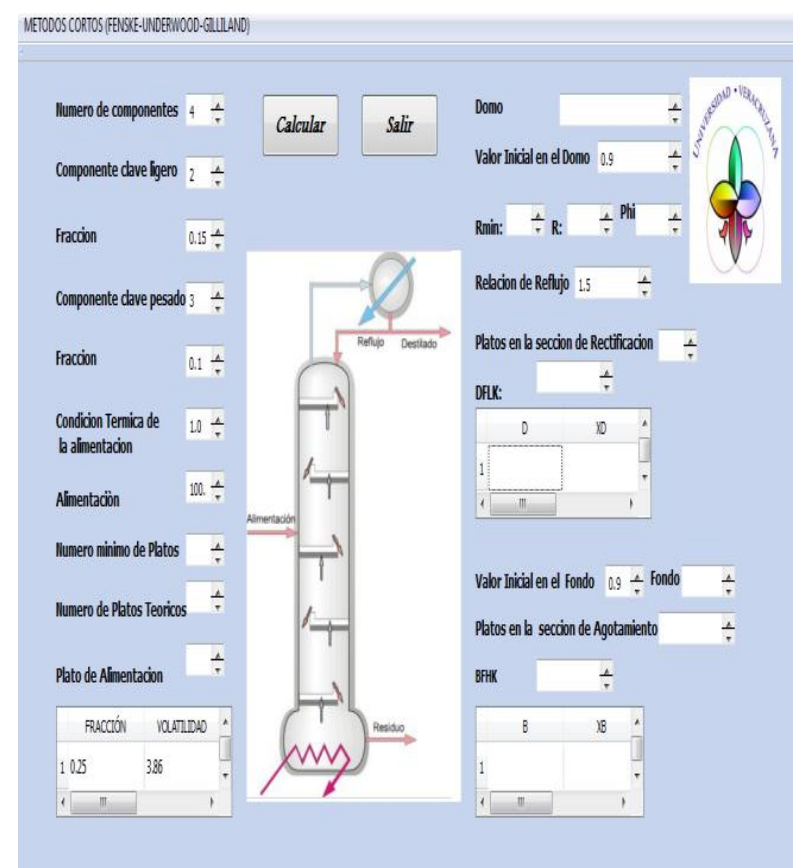

Figura. 4 Interfaz para el laboratorio virtual.

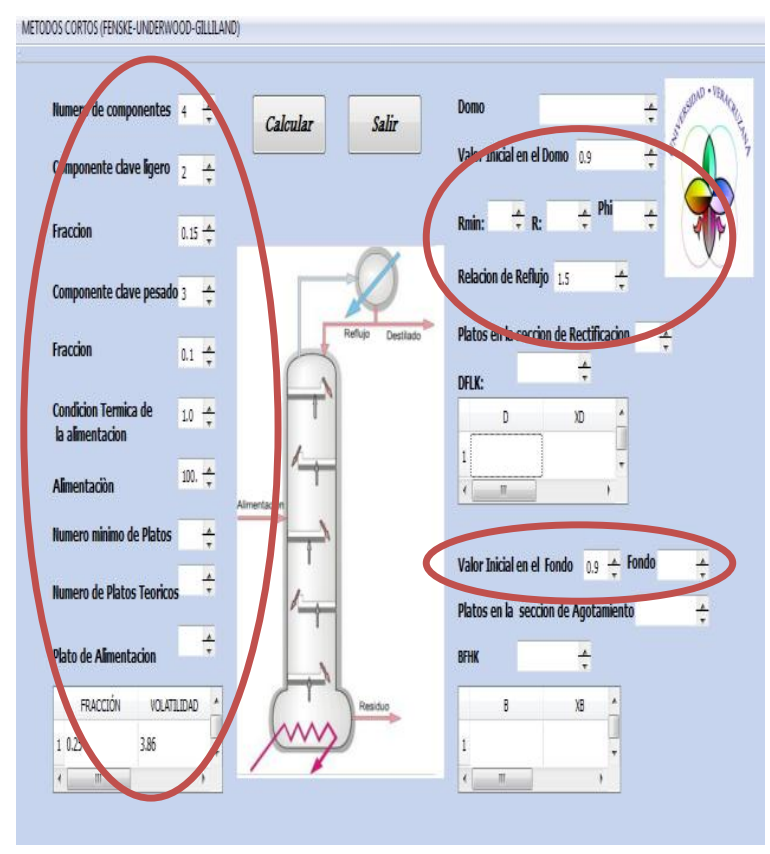

Figura. 5 Datos de entrada dados por el usuario.

Con el botón Calcular, Fig. 6, se ejecuta el programa y se muestran los resultados numéricos. Estos se pueden comparar con los datos obtenidos por medio de otros lenguajes de programación. Si ya no se desea realizar otros cálculos con el botón Salir se termina de utilizar la interfaz. 


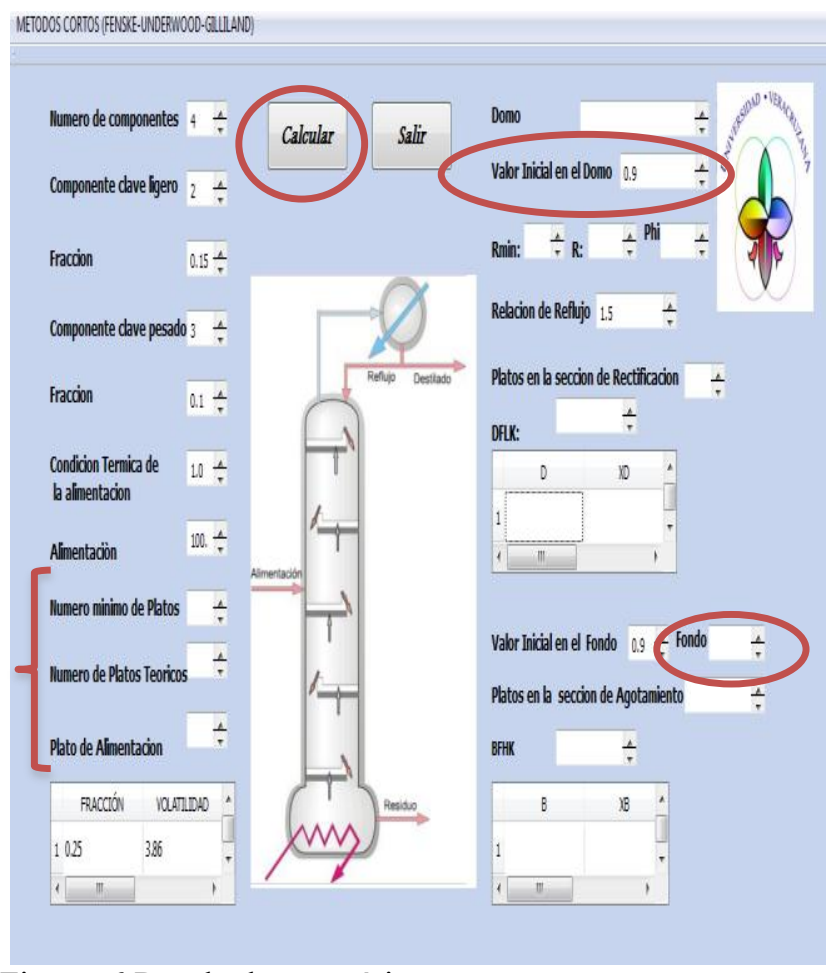

Figura. 6 Resultados numéricos.

A continuación se resuelve un ejercicio práctico aplicando la metodología descrita. En una destilación multicomponente la alimentación es un líquido saturado $(q=1)$ que tiene la composición y volatilidad, mostrada en la Tabla 1, (Chang, 1980). La razón de reflujo de operación es de 1.5 veces $R_{\min }$. Obtener una solución para la especificación $X_{B, L K}=0.15 ; R / R_{\min }=1.5 ; X_{D, H K}=$ 0.10 .

Tabla 1 Fracciones molares y volatilidades relativas de

\begin{tabular}{cccc} 
Componente & $\begin{array}{c}\text { Fracción } \\
\text { molar }\end{array}$ & $\begin{array}{c}\text { Volatilidad } \\
\text { relativa }\end{array}$ & $\begin{array}{c}\text { la } \\
\text { mez } \\
\text { cla. }\end{array}$ \\
\cline { 1 - 3 } 1 & 0.25 & 3.86 & $\begin{array}{r}\text { En } \\
\text { la }\end{array}$ \\
$2(L K)$ & 0.25 & 1.89 & $\begin{array}{r}\text { inter } \\
\text { faz }\end{array}$ \\
$3(H K)$ & 0.25 & 1.00 & $\begin{array}{r}\text { se } \\
\text { espe }\end{array}$ \\
4 & 0.25 & 0.65 & res
\end{tabular}

cifican las fracciones mol de los componentes clave pesado, que se espera obtener en el domo, y clave ligero a obtenerse en el fondo.

$$
X D(H K)=0.1000 \quad X F(L K)=0.1500
$$

Los valores iniciales de las distribuciones son:

$$
D F L K=0.9000 \quad B F H K=0.9000
$$

Después de 3 interaciones, los valores de distribución hallados son:

$$
D F L K=0.6025 \quad B F H K=0.8110
$$

A continuación se indica cuales son las cajas en las que se introducen los datos que son calculados o estimados por el usuario: número de componentes de la mezcla, clave ligero y pesado, fracciones mol, valores iniciales en el fondo y en el domo, etc., (Fig. 7); también, se indica en donde se muestran los resultados numéricos de la solución del problema (Fig. 8).

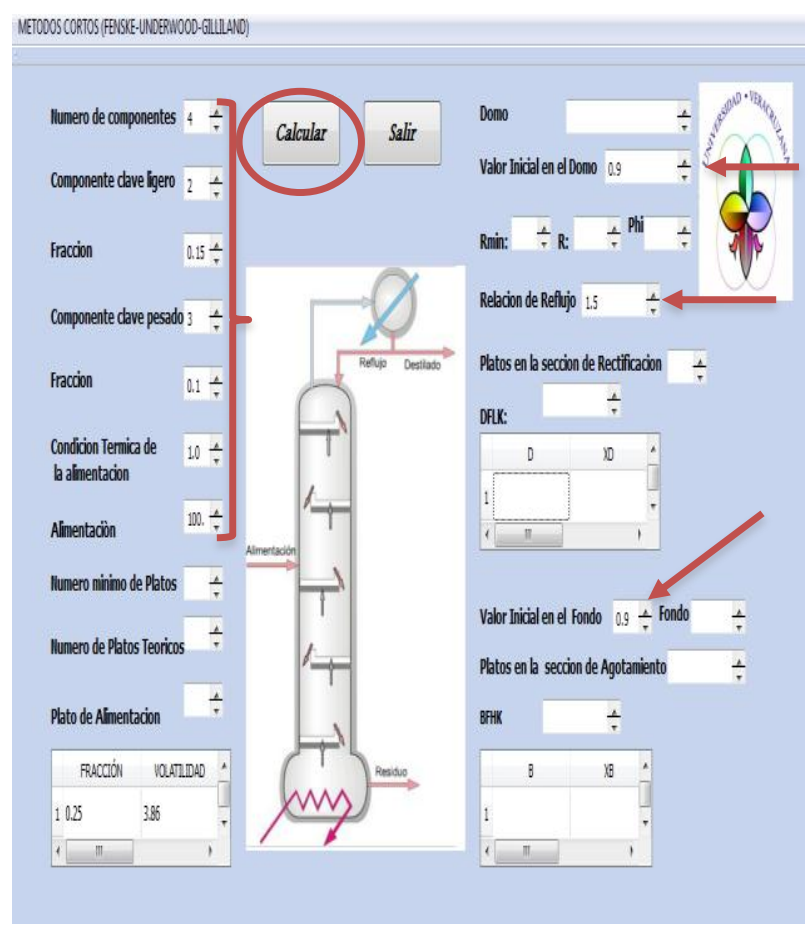

Fig. 7 Introducción de datos en la interfaz de usuario.

Se puede observar que con el uso de esta herramienta de software se alcanza una buena aproximación respecto a otros resultados presentados en la literatura.

A partir de estos resultados numéricos, considerando el número de platos teóricos y la posición del plato de alimentación se establecerá un modelo dinámico para la destilación multicomponente, considerando mezclas pseudobinarias. 


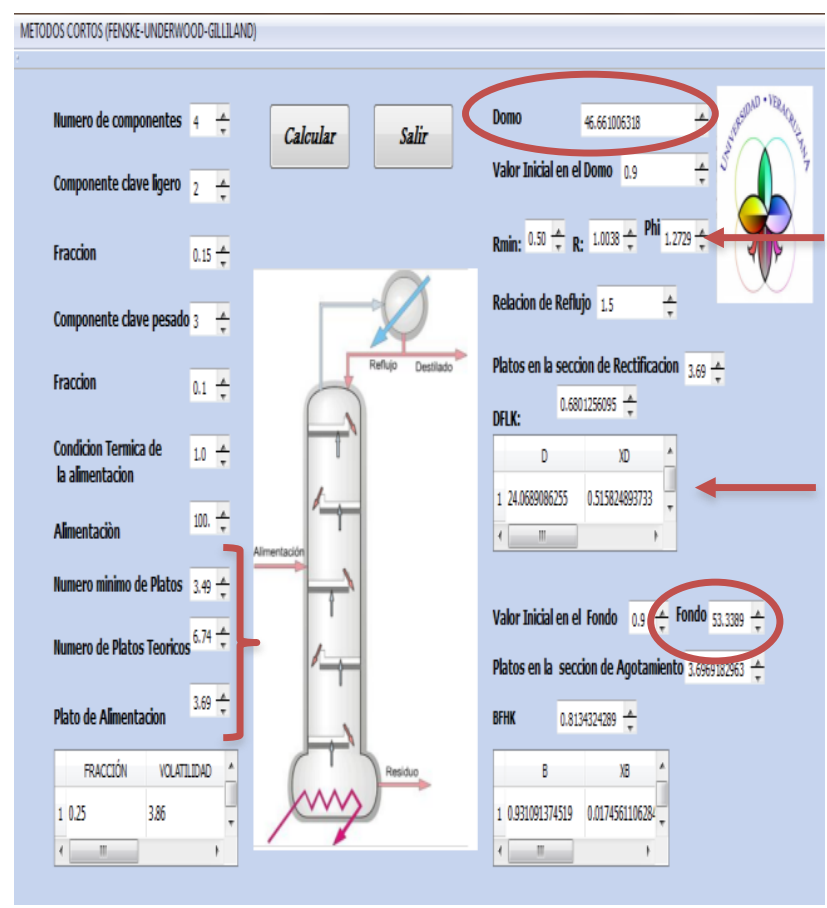

Fig. 8 Resultados numéricos.

La interfaz gráfica se modificará, para visualizar el perfil de comportamiento a lo largo de toda la columna, y el comportamiento dinámico de las concentraciones en cada etapa.

\section{CONCLUSIONES}

El programa desarrollado permite comprender el cálculo en separaciones de mezclas multicomponentes utilizando métodos cortos. Este software es capaz de calcular en forma rápida el número de platos teóricos y posición del plato de alimentación, el reflujo mínimo, así como las composiciones en el domo y el fondo de la columna con una eficiencia media del $89.5 \%$. Además, estos datos se pueden tomar como estimados iniciales para el cálculo con un método riguroso. La interfaz gráfica tiene como finalidad didáctica el uso en diseños de columnas de destilación multicomponentes para cálculos en estado estacionario y en el análisis dinámico del modelo establecido a partir de estos mismos, tambien es posible complementar la interfaz con módulos para control, estimación de parámetros y variables de estado; ademas de ser amigable al usuario lo que permite reafirmar los conceptos básicos en el estudio de procesos de separación.

\section{BIBLIOGRAFÍA}

King C.J. (1981). Separation Processes, McGrawHill, USA.

Kister H. (1992). Distillation Design, McGraw-Hill, USA.

Chang H.Y. (1980). Computer aids short-cut distillation design, Hydrocarbon Processing, August, pp. 79-82.

Dimitrios S.M.K. (1982). A short-cut method for multicomponent distillation, Chemical Engineering, March, pp. 83-86.

Schmitz Abe K.E. (1999). Modelado y simulación de una torre de destilación multicomponente. Tesis de Maestría en Ciencias, Instituto Politécnico Nacional. México.

Monroy-Loperena R. (2003). Convergence promotion in the Wang-Henke Tridiagonal Matrix Method, Canadian Journal of Chem. Eng., Vol. 81, No. 5, pp. 1092-1100.

Al-Tuwaim M.S., W.L. Luyben(1991), Multicomponent batch distillation. 3. Shortcut design of batch distillation columns, Industrial \& Engineering Chemistry Research, Vol. 30, No. 3, pp. 507-516.

Abad-Zárate E., J. Segovia-Hernández, S. Hernández, A. Uribe-Ramírez. (2006). Simulación de la columna de destilación Petlyuk usando el modelo de etapa en no equilibrio, Revista Mexicana de Ing. Quím., Vol. 5 (Supl 1), pp. 101-107.

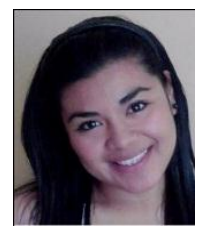

Dominguez Hernández Gloria desea agradecer especialmente a: FOMIX CONACYT-Veracruz, proyecto 109491. Los autores agradecen la colaboración de: Ing. Juan Leobardo Santiago Rosales e Ing. Martín García García, y de manear especial a el M. C. Miguel Vicente Teco Jácome de la Universidad Politécnica de Huatusco, Veracruz, México. Actualmente, Domínguez Hernández está con el CICATA-IPN, Unidad Altamira, en Altamira, Tamps., México. 